\title{
ON THE ESTIMATION OF URIC ACID IN URINE.
}

\author{
By F. Gowland Hopkins, B.Sc., F.I.C., Gull Research Student \\ at Guy's Hospital, London.
}

IN a paper read before the Royal Society last year, ${ }^{1}$ I described a method for the estimation of uric acid in urine, by means of saturation with ammonium chloride. Since the reading of that paper I have made considerable use of the method, under the most varying conditions; and it is the purpose of the present paper to give the results of this extended experience, and to describe fully the details of the process in a perfected form. After using it continuously for more than a year, with a large number of normal and pathological urines, I am strengthened in my belief that it is a process of very great accuracy. By its means uric acid may be estimated with an error of less than 1 per cent.; a degree of accuracy unattainable in the case of any of the other organic constituents of the urine.

\section{The Separation.}

The convenience of separating the acid as an ammonium salt is very great, as compared with that of its precipitation as the compound of a heavy metal, such as silver. The danger of reduction, which is so great in the latter case, is obviated; while the ammonium salt is easy to filter, and permits of the liberation of the free acid with great readiness. These are merits which belonged to the well-known Fokker Salkowski process, and if in the latter the uric acid underwent even approximately complete precipitation, there would have been little need to have sought further for a method of determining this important urinary constituent. Unfortunately the results obtained by the Fokker-Salkowski process require a very large correction for incomplete precipitation. In its most approved form, one is directed to add 30 milligrammes to the result obtained when working on 200 c.c. of urine. ${ }^{2}$

Now, as the product actually weighed in such a case will average some 100 milligrammes, it will be seen that the correction factor is most undesirably large. If it were really a constant, this would not, of

${ }^{1}$ Proc. Roy. Soc., 1892, vol. lii., p. 93.

2 Virchow's Archiv., 1876, Bd. Ixviii., p. 401. 
course, matter, but repeated experiments have convinced me that it is very far from being so.

Recognising the great convenience of an ammonium separation, I tried for a very long time, doubtless in common with other observers, so to modify the conditions of the Fokker-Salkowski process as to obtain more complete precipitation.

These experiments did not, however, guide me to the fact that a solution of the difficulty was to be found in pushing the addition of ammonium chloride to saturation point. $A$ priori, in fact, such a procedure would not have seemed convenient, or very feasible; though experience has shown it to be both. But, later, I was engaged in the study of the compounds of uric acid with mono-, di-, and trimethylamine, highly insoluble substances (as yet, I believe, undescribed), which I thought at the time might aid in this apparently elusive problem of uric acid estimation. It was with these bodies that I first noticed the effect of saturation with the hydrochloride of the base; upon which occurring, all uric acid, however previously combined in a solution, was thrown out as a compound of the particular base used.

Thereupon I harked back upon ammonia, and found that, in solutions of ammonium urate, saturation with ammonium chloride produced an immediate quantitative separation of the whole of the ammonium urate. A saturated solution of ammonium chloride is, in fact, a menstruum in which ammonium urate is absolutely insoluble. I also found that in solutions which contain the urates of mixed bases, it was only a question of time (and that a comparatively short time) for the uric acid to separate, in the same way, wholly in the form of ammonium urate. From urine the separation is always complete within two hours.

In the paper above referred to, I have given figures which show how completely the separation takes place when solutions of pure urates are concerned, and further results will be found below.

Leaving for the moment a discussion of the more difficult proof that in urine the precipitation is equally complete, it will be well, at this point, to consider the actual composition of the precipitate which one obtains in the case of human urine.

When a normal acid urine is saturated with perfectly pure ammonium chloride, the resulting precipitate, after washing with a saturated solution of the latter, yields, on incineration, a mere trace of ash, none of the mineral salts being carried down. Of the organic urinary constituents, other than uric acid, xanthin and certain pigments are the only ones that appear to be precipitated.

Xanthin is undoubtedly so precipitated, at any rate, from its solutions, in ammonium hydrate. There is every probability, therefore, that small quantities are present in the ammonium urate precipitate. If so, however, they are redissolved when the latter is decomposed by $\mathrm{HCl}$ in continuation of the process. I have prepared artificial mixtures of uric acid with small proportions of xanthin, and that although the 
latter is largely precipitated by ammonium chloride, the whole may be accounted for in solution when the uric acid is liberated. Hypoxanthin and creatinin are not precipitated; nor are any other organic constituent that I have been able to discover, with the exception of pigment.

Pigment.-The precipitate is always more or less pigmented, the usual affinity of uric acid and urates for urinary pigments being manifested. But the pigment is, under ordinary circumstances, extremely small in amount; and, fortunately, in normal urines and in the great majority of pathological ones, the pigment remains in solution in the $\mathrm{HCl}$ used to decompose the urate, the uric acid itself separating nearly white.

The precipitate produced by ammonium chloride shows a certain selective power in its pigmentation. Previous to the appearance of A. E. Garrods' paper on "Hæmatoporphyrin in Urine," 1 I had noticed that in certain pathological samples the absorption bands of acid hæmatoporphyrin were well marked in the filtrate from the uric acid liberated by $\mathrm{HCl}$. Since reading that paper I have made more careful observation, and have found the bands in a surprisingly large number of such filtrates. Comparing it in such cases with the phosphate method described by Garrod, I am inclined to think that the ammonium chloride precipitate is distinctly the more reliable as a means of separating hæmatoporphyrin.

In these cases, little or none of the pigment clings to the crystals of the liberated acid. It will be seen, therefore, that such substances as are likely to be present in traces as impurities in the urate precipitate are practically all removed by the subsequent treatment with hydrochloric acid.

Having now shown that there is nothing separated with the uric acid which will interfere with its subsequent estimation, it remains to prove that the acid is itself fully and completely precipitated by the ammonium chloride. If, after saturation, a urine be filtered when it has stood, say fifteen minutes, the filtrate will be clear at first, but after a short time it will begin to grow cloudy, and a further precipitate of urate will come down. If, however, the original sample be allowed to stand for a period which in no case need be longer than two hours, the filtrate will remain absolutely clear, even if it stand for weeks. To test directly such a highly saline solution as this filtrate for a trace of uric acid, would be impossible; but, by keeping it alkaline with carbonate of soda, while the ammonium salt is allowed to crystallise out, one may eventually obtain a solution from which ammoniacal nitrate of silver should precipitate even traces of uric acid. I have never been able to discover such a trace even when the filtrate from two litres of urine has been treated. Much more convincing than this, however, are the results of the following experiments:-

In Series 1 the uric acid was determined in each sample, while, to a

1 Joum. of Phys., 1892, vol. xiii., p. 598. 
second quantity of the same sample, a known amount of the pure acid was added, and the whole determined. Any disturbing factor in the precipitation would probably at any rate tell upon that added, as well as upon that already present; but it will be found that the increment estimated is always equal to the amount added.

Serics 1.

\begin{tabular}{|c|c|c|c|}
\hline Sample. & $\begin{array}{c}\text { Original quantity } \\
\text { milligrammes } \\
\text { per } 100 \text { c.c. }\end{array}$ & $\begin{array}{c}\text { Uric acid } \\
\text { dissolved in } \\
\mathrm{NA}_{2} \mathrm{CO}_{33} \\
\text { added in } 100 \mathrm{c.c.}\end{array}$ & Total found. \\
\hline I. Normal urine, . . & $52 \cdot 1$ & 20 & $72 \cdot 2$ \\
II. Normal urine, . . & $61 \cdot 0$ & 20 & $80 \cdot 8$ \\
III. Urine of pernicions & $57 \cdot 4$ & 30 & $88 \cdot 0$ \\
anmmia, . . & $89 \cdot 2$ & 10 & $99 \cdot 6$ \\
V. Fobrile urine, . . & $45 \cdot 3$ & 40 & $85 \cdot 4$ \\
\hline
\end{tabular}

In Series 2 urines of different characters were mixed in equal parts, the uric acid being determined in the original samples and also in the mixture. The result is always close to the calculated mean.

Series 2.

\begin{tabular}{|c|c|c|c|c|}
\hline Samples. & & $\begin{array}{l}\text { Containing } \\
\text { in milligrangmes } \\
\text { per } 100 \text { c.c. }\end{array}$ & $\begin{array}{l}\text { Calculated } \\
\text { mean. }\end{array}$ & Determined. \\
\hline $\begin{array}{l}\text { Normal urine, } \\
\text { Pernicious anæmia, }\end{array}$ &. & $\left.\begin{array}{l}40 \\
57\end{array}\right\}$ & $48 \cdot 5$ & $47 \cdot 6$ \\
\hline $\begin{array}{l}\text { Normal urine }(a) \text {, } \\
\text { Normal urine }(b), \text {. }\end{array}$ & $:$. & $\left.\begin{array}{l}88 \\
41\end{array}\right\}$ & $64 \cdot 5$ & $64 \cdot 0$ \\
\hline
\end{tabular}

In the case of a complex fluid such as urine, where the conditions are so largely unknown, it is difficult to see how the completeness with which any given constituent is separated can be tested directly, otherwise than by varying the conditions in some such way as was done in the above experiments.

As far as they go these results speak absolutely in favour of the accuracy of a process based on the ammonium chloride separation.

There remains the possibility of comparison with other processes. $\mathrm{By}$ far the most generally accepted of these is the Ludwig-Salkowski silver method.

In my previous paper ${ }^{1}$ I have shown that the process now described gives results agreeing very closely indeed with this standard method, and further trials have only gone to show that the more experience one has

1 Proc. Roy. Soc., loc. cit. 
with the really difficult procedure of the latter, and the better one learns to avoid the more fertile source of error it contains, the more nearly will the ammonium chloride method agree with it. I am certain that this will be the experience of any one who has opportunities of comparing the two.

\section{The Liberation of the Free Uric Acid.}

There are no difficulties connected with this. The urate precipitate needs only to be heated just to boiling point for a few moments with a fair excess of hydrochloric acid, when the decomposition will be found complete, the change from the flocculent urate to the heavy crystalline free acid being obvious. A practical point, however, may be mentioned here. When the uric acid crystals are filtered and washed, it is usual in all processes to measure the filtrate and washings together, and to make corresponding allowance for the solubility of uric acid in water. If pure uric acid be experimented on it will be found that the washwater, as a matter of fact, has no time to take up any appreciable quantity of the acid. Greater accuracy, therefore, is obtained if the filtered mother-liquor is measured before washing is begun, one milligramme being added to the final result for each 15 c.c. of motherliquor (vide infra).

\section{The Final Estimation.}

In the paper above referred to I have strongly recommended the use of standard potassium permanganate solutions, for the final determination of the liberated acid. I am able now to emphasise that recommendation with still stronger conviction.

In no analytical process where permanganate of potassium is used for the titration of organic bodies, is the reaction more determinate than in the case of uric acid. At a temperature of about $60^{\circ} \mathrm{C}$., the oxidation occurs in two definite stages-first a rapid, and almost instantaneous reaction takes place, followed by a further process which, at the temperature mentioned, is slow and gradual. The definite transition from one to the other of these stages enables one to perform an easy and accurate titration.

Sutton ${ }^{1}$ has previously suggested the use of potassium permanganate for estimations of uric acid, though its use in the analysis of urine was impossible till a satisfactory method of preliminary separation was available. It is perhaps surprising at first sight that uric acid, separated by any method direct from a fluid-like urine, should be sufficiently free from other organic matter, to be accurately titrated by permanganate solutions; but there is no doubt of the fact.

I describe below the exact method of titration which I have found the most satisfactory, but will here give a series of experiments on pure uric acid which are evidence at once of the completeness of the

\footnotetext{
1 Volumetric Analysis, 4th ed., p. 89.
} 
ammonium chloride separation and of the accuracy of permanganate titration. In each case the pure acid was dissolved in a minimum of sodium carbonate, made up to 100 c.c., precipitated by saturation with ammonium chloride, allowed to stand, and then titrated in exact accordance with the method described below for urines. The acid used had been purified by crystallisation as sulphate from strong sulphuric acid; that sold as pure being seldom reliable.

\begin{tabular}{|c|c|c|}
\hline $\begin{array}{c}\text { Pure Uric Acid } \\
\text { weighed. } \\
\text { Milligrammes. }\end{array}$ & $\begin{array}{c}\frac{\mathrm{N}}{20} \text { Permanganate } \\
\text { used. } \\
\text { C.C. }\end{array}$ & $\begin{array}{c}\text { Caiculated from } \\
\text { Titration. } \\
\text { Milligrammes. }\end{array}$ \\
\hline 20 & 5.45 & $20 \cdot 44$ \\
30 & $8 \cdot 10$ & $30 \cdot 37$ \\
45 & 12.00 & $45 \cdot 00$ \\
55 & $14 \cdot 70$ & $55 \cdot 12$ \\
120 & $32 \cdot 40$ & $121 \cdot 50$ \\
\hline
\end{tabular}

It should be here stated that the results given in the above experiments on urine (p. 454) were also obtained by titration, and not by weighing, so they are, therefore, evidence in favour of the titration as well as of the separation itself. These titrations were performed with onetwentieth normal permanganate ( 1 c.c. $=00375 \mathrm{grm}$. uric acid), but the end-point is so clear and definite that one-fiftieth normal $(1$ c.c. $=0015)$ can be used, and is convenient when small quantities of urine are employed as in clinical work (vide infra). The tendency of error is at first towards slightly high results by overshooting the end-point, but very little practice enables one to recognise this the moment it appears. By titrating the uric acid separated from urine, instead of weighing it, one gains, in addition to much greater despatch, certain other advantages which make for accuracy. For instance, even moderately good samples of ammonium chloride may contain sufficient insoluble silicious matter to affect slightly the final weighing; such impurity, present to the extent of 1 in 30,000 , would mean about a milligramme added to the final result (about 30 grammes being necessary to saturate 100 c.c. urine), so that special purification of the chloride is necessary when the determination is made by accurate weighing.

In titration, such insoluble matter does not, of course, affect the result. Moreover, in the process of washing, first the urate and afterwards the free acid from their respective filters, if the filters happen to be of loose texture, paper fibres having appreciable weight may be got into the final product; but cellulose is not appreciably oxidised by permanganate during the progress of the first and instantaneous stage of uric acid oxidation. Finally, I believe that any slight amount of pigment that may cling to the final product also fails to affect the permanganate during the rapid titration. This would seem to be proved by the fact 
(vide supra) that when uric acid is artificially added to a highly pigmented urine the increment as titrated is not in excess of the added quantity, although the latter undoubtedly brings down its share of pigment.

These details may seem to deal with minutix, but $I$ believe that such may affect the result to an extent which falls outside the limits of necessary error. If titration results are compared with weighings on the same sample, the difference is always under 1 per cent., the latter being usually the higher.

\section{Practical Detalls of the Process as applied to Urines, Normal and Pathological.}

a. In normal urines without deposit.-(1) To 100 e.c. of the sample, powdered ammonium chloride is added till practical saturation is obtained; about 50 grammes are necessary, but there is little need to weigh out the salt. When a small quantity of the chloride is seen to remain undissolved, even after brisk stirring at intervals of a few minutes, saturation is sufficiently complete. As the temperature of the urine again rises, from the depression due to the process of solution, any residual crystals will for the most part dissolve, but there will be no need to add more.

(2) After having stood for two hours, if possible with occasional stirrings, to promote subsidence, the precipitate produced is filtered through a thin filter-paper, and washed three or four times with a saturated solution of ammonium chloride. The filtrate should remain perfectly clear and bright.

(3) With a jet of hot distilled water, the urate, which will be somewhat pigmented, is now washed off the filter into a small beaker, and heated just to boiling, with an excess of hydrochloric acid. It is then allowed to stand for the uric acid to separate out completely. Two hours is sufficient for this, if the liquid is cooled. The acid is then filtered off and washed with cold distilled water. The filtrate should, however, be measured before the washing is begun, and 1 milligramme added to the final result for each 15 c.c. of liquid present. This need never be more than from 20 to 30 c.c.

(4) The acid is now again washed off the filter with hot water, warmed, with the addition of sodium carbonate, till dissolved, and the solution then made up to 100 c.c. Being transferred to a flask of sufficient capacity, it is mixed with 20 c.c. of strong pure sulphuric acid, and immediately titrated with one-twentieth normal potassium permanganate solution. The latter should be added slowly towards the end of the reaction, the close of which is marked by the first appearance of a pink colour, which is permanent for an appreciable interval. Previously, the disappearance of the colour will have been quite instantaneous (vide supra). The flask should, of course, be agitated throughout the operation. 
The standard solution is made by dissolving 1.578 grammes of pure potassium permanganate in a litre of water. As stated above, 1 c.c. of this is equal to 00375 grammes of uric acid. The addition of 20 per cent. of sulphuric acid to the solution produces a temperature suitable to the reaction, and no thermometer need be used.

b. In acid urines with deposit.-If the deposit be only uric acid or urates, there is but little danger in neglecting it. Add the ammonium chloride to bring down such uric acid as remains in solution, and treat the whole, precipitate plus deposit, as above. The deposit most likely to lead to error would be one of cystin. If this be suspected, a few drops of ammonia should be added and the urine warmed. The chloride of ammonium may be added while the sample is still warm (vide (c) infra).

c. Alkaline urines. - If the deposit of phosphates be very large, it may be well to filter them off, and wash with hot water; otherwise they may be neglected. The ammonium urate comes down on saturation with chloride much more rapidly in alkaline urines. ${ }^{1}$ The only objection to adding free ammonia in all cases is the precipitation of phosphates (vide, however, loc. cit.) and the fact that the urate precipitate appears to absorb more pigment from the alkaline urine. Continue the process as in $a$.

d. Albuminous urines.-Ammonium chloride has fortunately but a slight precipitatory power for albumen, as compared, e.g., with the sulphate; and I believe that by a somewhat longer digestion than usual with hydrochloric acid, when the urate precipitate is decomposed, the uric acid crystals are to be obtained quite free from proteid. A good excess of hydrochloric acid should be present to form acid albumen.

e. Highly pigmented urines.-Sometimes, though very rarely, the uric acid crystals may be so much pigmented as to suggest that an error in titration might result. In such cases the original urate precipitate may be decomposed. in the presence of alcohol. After washing the precipitate off the filter, rectified spirit equal in bulk to the water present is added, and, after acidification, the liquid is cautiously heated to boiling in the usual way, and then, with a clock glass in the beaker, it is digested for some time in the water bath. The separated crystals should be well washed. In the urines which contain bile, the biliary pigment may come down in considerable quantity, but, in spite of this, the ultimate error appears to be small.

The whole process, as set forth above under $a$, may be tedious in description; but in practice it will be found very easy to carry out, and very much more rapid than any other process, the accuracy of which is at all generally accepted.

\section{Shortened Process for Clinical Work.}

I shall close this paper with an account of a modification of the method for clinical purposes, which I have recently compared with the full process as described above. Taking into consideration the extreme facility of the abbreviated method, its results are surprisingly accurate. I have previously shown ${ }^{2}$ that the original ammonium urate precipitate may be directly titrated without any considerable error, and this fact is taken advantage of in the following process.

1 Proc. Roy. Soc., loc. cit., p. 96.

${ }^{2}$ Guy's Hospital Reports, 1891, vol. xlviii., p. 299. 
Twenty cubic centimetres only of urine are used, and the precipitate produced is titrated with a weaker permanganate solution, one-fifth normal. The following modifications are necessary:-

A filter is prepared by packing a small plug of glass-wool tightly into the neck of a small glass funnel. The 20 c.c. of urine, after saturation with ammonium chloride, and standing for the usual two hours, is filtered through this filter, and washed, not with a solution of the chloride, but with a saturated solution of the sulphate of ammonium. This is to remove chlorides which interfere with a direct titration. The chloride is much superior to any other salt of ammonium as a precipitant, and the excess is easily washed away by the sulphate when a glass-wool filter is used. No wash-bottle need be used for the sulphate solution; it may be run on to the filter from a pipette. After washing, the glass plug, together with the entangled precipitate, is bodily transferred to a small flask, boiled up with a little sodium carbonate solution, and titrated in precisely the same way as is the final product in the full process; the dilution, acidification, etc., being made proportionate. It is as well to wash the filter before use with a little of the sulphate solution, to remove loose pieces of glass fibre, otherwise these latter may lead one to think that the precipitate is coming through. The weak permanganate is best made from time to time by diluting stronger solution; it keeps very well however $(1$ c.c. $=\cdot 0015$ uric acid).

When one remembers the extreme sensitiveness of permanganate salts to all forms of organic matter, it may seem surprising that a product separated from such a fluid as urine could thus be titrated directly with any approach to accuracy; but the following figures speak for themselves. The maximum error introduced by the xanthin and pigment present in the precipitate seems to be under 5 per cent. Such a process as this has a great advantage over a method like that of Haycraft, in that one is titrating the uric acid itself, and not an associated substance like silver, the percentage of which in the precipitate seems to be much a matter of doubt.

\begin{tabular}{|c|c|c|}
\hline Sample. & $\begin{array}{c}\text { Milligrammes per } \\
\text { 100..c. determined } \\
\text { by full process. }\end{array}$ & $\begin{array}{c}\text { The same calculated } \\
\text { from abrevisted } \\
\text { process. }\end{array}$ \\
\hline 1 & $51 \cdot 5$ & $53 \cdot 2$ \\
2 & $64 \cdot 0$ & $66 \cdot 5$ \\
3 & 40.7 & $41 \cdot 1$ \\
4 & $34 \cdot 4$ & $35 \cdot 5$ \\
5 & $38 \cdot 0$ & $39 \cdot 6$ \\
6 & 6.54 & 46.9 \\
\hline
\end{tabular}

